\title{
In der neuen Heimat angekommen
}

\section{Matthias Scholer}

Chefredaktor SÄZ

\author{
Rund ein Drittel der Ärztinnen und Ärzte in der Schweiz kommt aus dem Ausland. \\ Weshalb haben sie sich für ein Leben hierzulande entschieden? Welches sind kultu- \\ relle Stolpersteine, die es zu umschiffen gilt? Und was halten sie von der Schweizer \\ Gesundheitspolitik? Diesen und weiteren Fragen möchten wir in der neuen, in \\ loser Folge erscheinenden Artikelserie «Grüezi Schweiz» nachgehen. In dieser Aus- \\ gabe stellen wir Tim Klose vor, dem es in der Schweiz «den Ärmel reingenommen \\ hat».
}

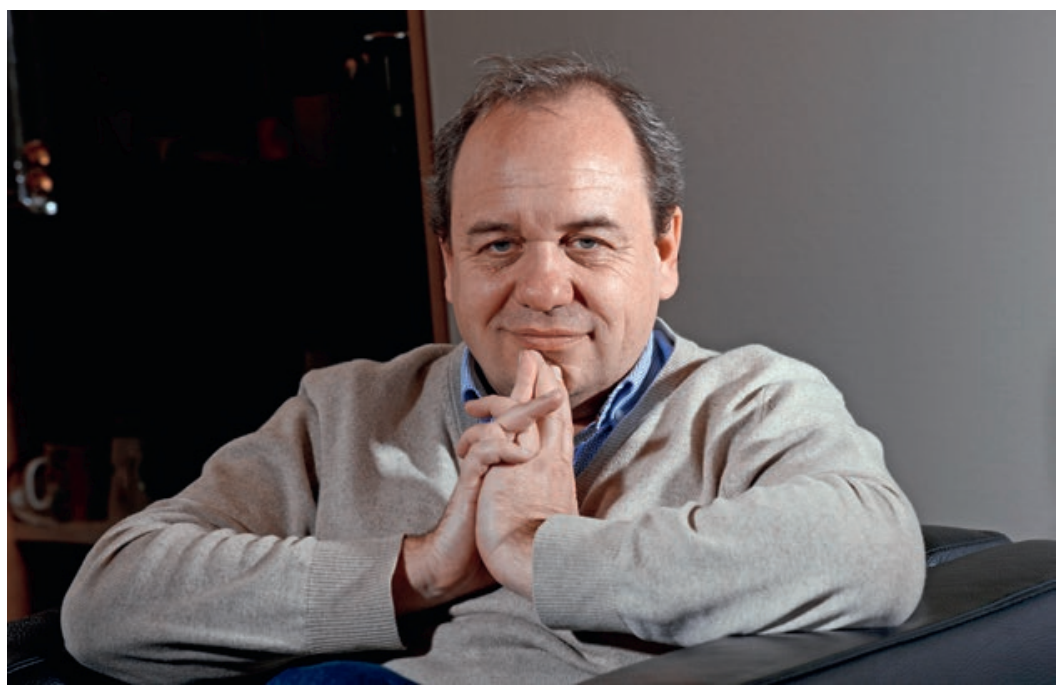

Tim Klose wagte vor kurzem den Schritt in die Selbständigkeit.

Gleich zu Beginn unseres Gesprächs bittet mich Tim Klose, im Dialekt zu sprechen. Etwas, das er auch bei seinen Patientengesprächen bevorzugt. Denn: «Als Psychiater bekomme ich mehr Emotionalität mit, wenn sich meine Patientinnen und Patienten in Mundart ausdrücken.»

Kloses Weg in die Schweiz führte über Jerusalem. Hier absolvierte er sein Fremdjahr auf der chirurgischen Abteilung eines grösseren Spitals. «Diese Zeit war in mehrfacher Hinsicht ein vielschichtiges Spannungsfeld", erinnert sich der gebürtige Stuttgarter. Einerseits arbeitete er als Deutscher, dessen Grosseltern «zumindest möglicherweise als Wähler den Nationalsozialismus unterstützten», in einer jüdischen Einrichtung. Andererseits fiel sein Einsatz in eine Zeit, in der Bombenattentate auf Busse und Märkte zur Tagesordnung gehörten. «Hier lernte ich, wie man mit einem unerwartet grossen Patientenaufkommen und begrenzten
Ressourcen umgehen muss», erzählt Tim Klose. Und: "Auch einige Monate nach den Anschlägen kamen viele Bombenopfer zu Kontrolluntersuchungen in die Sprechstunde. Dabei wurde ich auch mit unterschiedlichen psychiatrischen Problemstellungen konfrontiert.» Trotz Terror und fordernder Arbeit gab es Zeit und Raum für das Schöne: «Ich verliebte mich im Spital in eine Schweizerin.»

\section{Eine tiefe Zäsur}

Nach dem Praktikum in Israel folgte eine Zeit des Pendelns zwischen Berlin und der Schweiz, bis sich der junge Arzt entschloss, seine Facharztausbildung im Bereich Psychiatrie und Psychotherapie hierzulande zu absolvieren. «Aufgrund des Ärztemangels fand ich recht schnell meine erste Stelle in der Klinik Littenheid», sagt Tim Klose. Diese Stelle gefiel dem angehenden Psychiater nicht nur dank der flachen Hierarchie: «In der Schweiz ist die Weiterbildung sehr gut strukturiert.» Dies kam ihm, der gerne mal den theoretischen Teil der praktischen Arbeit mit den Patienten unterordnet, zugute.

Doch nicht alles lief nach Plan. Die Beziehung ging in die Brüche. Ein tiefer Einschnitt in Kloses Leben, der ihn kurzzeitig sogar suizidal werden liess. Im Nachhinein sieht er es positiv: «Diese Erfahrung hilft mir Patienten zu verstehen, die in einer für sie ausweglosen Situation gefangen sind.»

\section{Die Medizin der Zukunft}

Nach der persönlichen Krise war sich Tim Klose nicht mehr sicher, ob er in der Schweiz bleiben wollte. Er setzte deshalb seine Weiterbildung mit einem Jahr auf der Neurologie in der Rheinfelder Klinik Schützen fort, 
um die Voraussetzung zu erfüllen, um in seinem Heimatland Deutschland «als Nervenarzt arbeiten zu dürfen». Doch es kam anders: "Mir hat es den Ärmel reingenommen, wie man hier so schön sagt, und ich entschied mich, in der Schweiz zu bleiben.» Die für die Facharztausbildung obligaten zwei Jahre in einer ambulanten psychiatrischen Institution absolvierte Klose in Oerlikon und Bülach und wurde anschliessend 39-jährig Chefarzt im Spital Affoltern. Er erinnert sich gerne an die für ihn «sehr spannende» Zeit, in diesem kleinen, aber sehr diversen Regionalspital: «Die Spitalleitung entschied sich schon früh, eine Palliativabteilung aufzubauen, und integrierte ab 2004 sogar eine psychiatrische Abteilung mit zwanzig Betten.» Den niederschwelligen Beizug von Psychiaterinnen und Psychiatern sieht Tim Klose denn auch als Modell für die Medizin der Zukunft. «Die Psychiatrie ist ein sehr integratives Fach, bei dem der Mensch und nicht die Krankheit in Vordergrund steht. Wenn schon beim Morgenrapport eine Psychiaterin, ein Psychiater anwesend ist und sich frühzeitig bei Fällen der medizinischen oder chirurgischen Abteilung einbringen kann, wirkt sich dies schlussendlich positiv auf die Versorgungsqualität eines Spitals aus», ist Klose überzeugt. In diesem Punkt sieht der Psychiater denn auch den grössten Aufholbedarf der Schweiz. Die meisten psychiatrischen Kliniken wurden hierzulande ausserhalb von Ortschaften oder in Kurlagen errichtet, was schliesslich zu einer Trennung von der Akutversorgung führt. Eine Zusammenführung der ambulanten und stationären Versorgung wäre auch der Entstigmatisierung psychischer Erkrankungen dienlich. Und: «Die Psychiatrie hat nach 150 Jahren akademischer Tradition einige Werkzeuge zu bieten, die allen Patienten zugute kommen können.» Klose engagiert sich auch standespolitisch, damit die künftigen psychiatrischen Assistenzärztinnen und -ärzte die bisher strenge Trennung zwischen stationär und ambulant nicht mehr wie bisher mühsam überbrücken müssen, sondern integrativ gleichzeitig in ambulanter, teilstationärer und stationärer Behandlung ausgebildet werden können, entsprechend den Patientenpfaden.

\section{Der Schritt in die Selbständigkeit}

Die Zukunft kleiner Spitäler ist ungewiss. Dies war denn auch der Grund, weshalb sich Tim Klose entschloss, den Schritt in die Selbständigkeit zu wagen,

Für unsere Serie "Grüezi Schweiz» suchen wir ausländische Ärztinnen und Ärzte, die uns einen Einblick in ihr Leben und ihren Berufsalltag gewähren. Wir freuen uns über eine Kontaktaufnahme: matthias.scholer[at]emh.ch nachdem er geeignete Räumlichkeiten in Zürich fand. Da sich die Praxis im Gebäudekomplex des ehemaligen Spitals «Sanitas» befindet, das mittlerweile nach Kilchberg umgezogen ist, gehört eine grosse Parkanlage dazu. Einen kleinen Teil davon darf Tim Klose allein nutzen. «Hier biete ich im Sommer Gespräche im Grünen an. Meine ganz eigene Form einer Gartentherapie», erzählt der Arzt lachend. Dass er in der Schweiz angekommen ist, zeigt sich auch in der Einbürgerung im Jahr 2013: «Ich wollte hier nicht nur Steuern bezahlen, sondern auch abstimmen können.»

\section{Sich auf eine andere Mentalität einlassen}

Würde Tim Klose seinen deutschen Kolleginnen und Kollegen raten, in der Schweiz Fuss zu fassen? «Ja, denn man kann in der Schweiz freier arbeiten und muss sich im Vergleich zu Deutschland mit viel weniger Administration rumschlagen", fasst der Psychiater die beruflichen Vorteile grob zusammen. Davon profitieren vor allem die Patientinnen und Patienten, da diese im Vergleich zu Deutschland meist ohne grössere Wartezeiten mit einer Psychotherapie beginnen können. Selbstverständlich müsse man sich bewusst sein, dass es zwischen den Ländern Mentalitätsunterschiede gibt. "Aber die gibt es schliesslich auch innerhalb Deutschlands», relativiert Klose. Die Frage, ob er während seiner Laufbahn in der Schweiz aufgrund seiner Herkunft je negative Erfahrungen seitens der Patienten gemacht hat, verneint er umgehend. Nur während seiner Tätigkeit an der Klinik Littenheid gab es Zuweiser, die sich bei seinen Vorgesetzten erkundigten, weshalb ihre Patienten von einem Ausländer und nicht einem Schweizer betreut würden. Auch wenn Tim Klose zwischenzeitlich voll und ganz in der Schweiz angekommen ist, wurde ihm während des Corona-Lockdown schmerzlich bewusst, wie sehr wir uns an die offenen Grenzen und das Zusammenleben innerhalb Europas gewöhnt haben: «Ich konnte während diesen Monaten meine in Stuttgart lebenden Eltern nicht besuchen.» Klose erlebte damit am eigenen Leib, was die Isolation von sozialen Kontakten bei Menschen auslösen kann. Eine weitere Selbsterfahrung, von der Kloses Patientinnen und Patienten profitieren können.

\section{Bildnachweis}

Paolo Foschini | DeinAdieu.ch

Pour notre rubrique «Bonjour la Suisse», nous recherchons des médecins étrangers pour nous raconter ce qu'ils pensent de leur existence et de leur quotidien professionnel. C'est avec plaisir que nous vous lirons: matthias.scholer[at]emh.ch 